\title{
Insertion Loss and Misalignment Tolerance in Multimode Tapered Waveguide Bends
}

\author{
Ioannis Papakonstantinou, Student Member, IEEE, David R. Selviah, Member, IEEE, and \\ Kai Wang, Student Member, IEEE
}

\begin{abstract}
Experimental measurements of laterally tapered multimode waveguide bends fabricated photolithographically on FR4 printed circuit board establish that the product of the mean insertion loss (in linear units) and the mean source misalignment tolerance is a constant which depends only on the taper ratio TR (input width/output width) and not on the radius of curvature $R$. The minimum loss of $0.78 \mathrm{~dB}$ occurs in the special case of $\mathbf{T R}=0.8$, $R=14 \mathbf{~ m m}$. Together, these form waveguide layout design rules for board-to-board and chip-to-chip optical interconnects.
\end{abstract}

Index Terms-Multimode polymer waveguide, optical printed circuit board, spotsize converter, tapered waveguide bend.

\section{INTRODUCTION}

$\mathbf{T}$ APERED waveguides have been shown to facilitate the coupling of light from a laser source into a waveguide or fiber in single-mode systems [1]. Similarly, tapered polymer waveguides have been used in multimode (MM) systems [2]. Cost requirements in MM systems mean that laser-to-waveguide and waveguide-to-photodetector alignment must be passive. For lowest cost, tight alignment tolerances must not be required so the coupling loss should be independent of large relative misalignments. Wide straight waveguides give large input misalignment tolerances for small aperture lasers, but at their output they might be larger than the active area of the photodetectors, which has to be kept small to minimize capacitance, causing excess loss and modal noise. A large input aperture waveguide is needed which tapers down in width to a narrow output aperture, but tapered waveguides can be lossy unless they taper down slowly or adiabatically, to minimize coupling between propagating and radiating modes; so, they must be long. In current electronic backplane designs, adjacent connectors are placed 1 in from each other so waveguides from one connector directly emerging in the direction of the next adjacent connector have to bend almost immediately to avoid the adjacent connector and there is not enough space for a long straight taper. However, [3] showed that the optimum radius of curvature for the multimode waveguides usually employed in

Manuscript received January 22, 2008; revised March 15, 2008. This work was supported in part by the U.K. DTI, in part by the U.K. EPSRC, in part by Storlite GR/S28136/01 under an EPSRC Doctoral Training Award, and in part by EPSRC I $e$ MRC OPCB. The work of I. Papakonstantinou was supported by Xyratex Technology, Ltd.

The authors are with the Department of Electronic and Electrical Engineering, University College, London, London WC1E 7JE, U.K. (e-mail: d.selviah@ee. ucl.ac.uk).

Color versions of some of the figures in this letter are available online at http:// ieeexplore.ieee.org.

Digital Object Identifier 10.1109/LPT.2008.923744

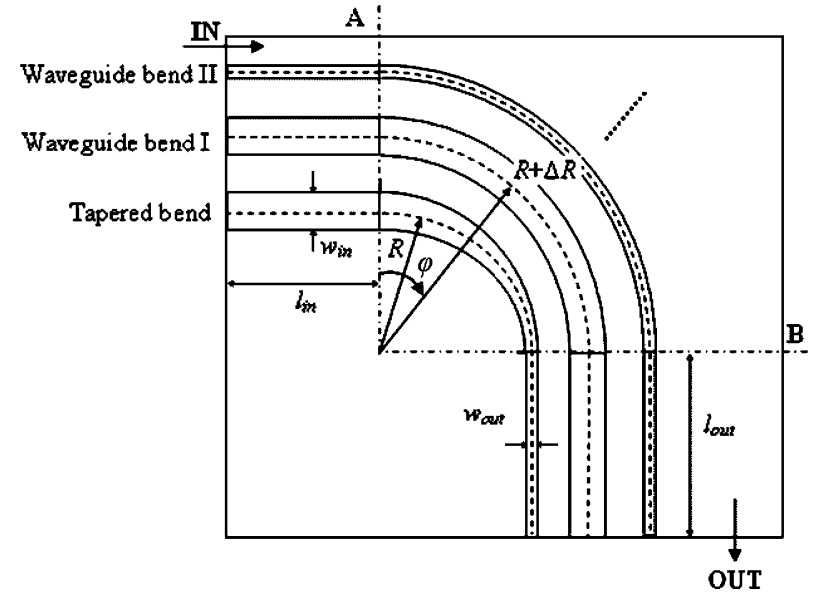

Fig. 1. One basic group of waveguiding components. From smaller to larger radii, each group contained one tapered bend, one waveguide bend with width $w_{\text {in }}$, and one waveguide bend with width $w_{\text {out }}$.

optical backplanes is $\sim 15 \mathrm{~mm}$ and this only just permits a $90^{\circ}$ waveguide bend between two adjacent connectors.

A solution, which can result in a more compact device, is to combine a taper and a bend in a single component, the tapered bend [4]. A thorough set of design rules for such a component based on experimental measurements remains to be established. This letter carries out numerous experimental measurements to establish design curves showing the tradeoff between the insertion loss and source misalignment tolerance for tapered bends as a function of radius of curvature and taper ratio (TR), which is the ratio of the input to the output width.

\section{INSERTION LOSS MEASUREMENTS}

\section{A. Tapered Waveguide Bend Description}

A schematic diagram of one group of polymer waveguides used in our experiments is shown in Fig. 1. The waveguide with the smallest radius $R$ is the tapered waveguide bend, which consists of a straight input waveguide, a $90^{\circ}$ tapered bend, and an output straight waveguide. The width of the input section is $w_{\text {in }}=50 \mu \mathrm{m}$, corresponding to standard multimode waveguide width for optical backplane applications, and its length $l_{\text {in }}=11.5 \mathrm{~mm}$. The tapered bend, which follows, transforms the waveguide width from its initial value $w_{\text {in }}$, to its final value $w_{\text {out }}$, changing the waveguide's direction by $90^{\circ}$. The width of the tapered bends varies linearly with respect to the polar angle $\phi$. Finally, the output straight waveguide has width $w_{\text {out }}$ and length $l_{\text {out }}=24.5 \mathrm{~mm}$. The output widths of the tapered bends were $w_{\text {out }}=10,20,25,30$, and $40 \mu \mathrm{m}$, giving corresponding 
taper ratios $\mathrm{TR}=0.2,0.4,0.5,0.6$, and $0.8,\left(\mathrm{TR}=w_{\text {out }} / w_{\text {in }}\right)$, and they were all $50 \mu \mathrm{m}$ thick.

The adjacent component with radius $R+\Delta R$ is an input straight — bend — output straight waveguide cascade (waveguide bend I). All three waveguides in this case have the same width $w_{\text {in }}=50 \mu \mathrm{m}$. Waveguide bends with $w_{\text {in }}=w_{\text {out }}=50 \mu \mathrm{m}$ can also be considered to be tapered bends with TR $=1$. The next component with radius $R+2 \Delta R$ is a second waveguide bend, where all of the waveguides have width $w_{\text {out }}$ (waveguide bend II). The separation between two successive waveguide optic axes is $\Delta R=250 \mu \mathrm{m}$. The mask was designed to host multiple such nested groups of three waveguides. The first group had $\mathrm{TR}=0.2$ and started from $R=5 \mathrm{~mm}$, followed by a second concentric group with TR $=0.4$ starting at $R=6 \mathrm{~mm}$, a third with TR $=0.5$ at $R=7 \mathrm{~mm}$, and so on until all five values of TR had been used, after which they were used again in the same order and so on with the last group starting at $R=34 \mathrm{~mm}$. There were six waveguide groups for each value of TR having different radius of curvature $R$. The waveguides were fabricated photolithographically on an FR4 printed circuit board (PCB) [3], using Truemode TM Acrylate polymer with core refractive index $n_{\text {core }}=1.556$ and cladding refractive index $n_{\text {clad }}=1.5264$.

\section{B. Insertion Loss Measurements}

Light from an 850-nm VCSEL was launched into a standard $50 / 125 \mu \mathrm{m}$ step index multimode fiber with numerical aperture $\mathrm{NA}_{\text {fiber }}=0.2<\mathrm{NA}_{\mathrm{wg}}=0.302$. After mode scrambling and checking that the fiber modes were almost fully filled, the fiber was aligned and butt-coupled to one of the waveguides on the PCB with the aid of three axis submicron motorized stages. Light from the waveguide output was spatially filtered through a $100-\mu$ m-diameter circular pinhole, to exclude much of the light traveling through the cladding, before being collected by an integrating sphere photodetector (PD). Index matching fluid ( $n=1.555$ at $850 \mathrm{~nm}$ ) was applied to both fiber-waveguide and waveguide-PD interfaces.

The calibrated insertion loss $P c a l$ as a function of the radius of curvature $R$, for the tapered bends, is shown in Fig. 2. Pcal is defined as the power measured at the end of one of the tapered bends minus the power measured at the end of the waveguide bend I of width $w_{\text {in }}$ in the same group (Fig. 1). Since the radius of the tapered and the waveguide bend I are approximately the same, this calibration step removes the coupling, transition, radiation, and propagation loss [3]. So, the calibrated insertion loss is the excess loss that a tapered bend exhibits, compared to the insertion loss of a simple waveguide bend of the same input width. This excess loss is due to the taper. Fig. 2 shows that irrespective of $R$, the insertion loss for the tapers with higher TR is always less than the insertion loss for the tapers with lower TR as expected, since as the output waveguide width reduces the higher order modes gradually move beyond cutoff and radiate giving higher loss. For TR $=0.2$ and $\mathrm{TR}=0.4$, the insertion loss gradually reduces as $R$ increases, while for the rest of the TR the insertion loss appears to be independent of $R$. One possible explanation is that as the radius reduces, the field from the input straight waveguide excites more strongly the high-order local modes of the tapered bend [5]. Since the low TR tapers

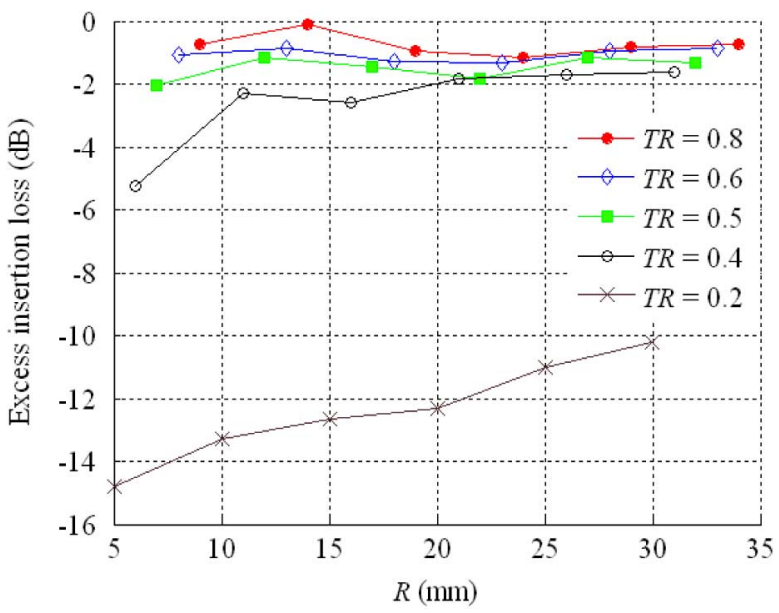

Fig. 2. Excess insertion loss as a function of radius $R$ of curvature for the tapered bends on the wafer for all available TR. $\left(\mathrm{TR}=w_{\text {out }} / w_{\text {in }}.\right)$

tend to filter out high-order modes more strongly, as these modes go beyond cutoff a significant amount of the power in the waveguide is lost which depends on the radius of curvature. For large TR $\geq 0.5$ though, the same effect is not as important since the output width $w_{\text {out }}$ appears to be large enough to support almost all of the excited tapered bend modes for any $R$. The tapered bends with $\mathrm{TR}=0.2$ have high insertion loss $>11 \mathrm{~dB}$ for all $R$ and, therefore, they are not suitable for use in a practical OPCBs where power budget is an important issue. In contrast, tapered bends in the range $0.5<\mathrm{TR}<0.8$ have excess loss, which is always $<2 \mathrm{~dB}$ for any radius, and so they might be considered to be used. In terms of insertion loss, the best tapered bend design is that for TR $=0.8$, with average insertion loss among the six measurements of $0.75 \mathrm{~dB}$. If that taper is too slow, then $\mathrm{TR}=0.6$ could be used at the expense of an increased average loss of about $1.1 \mathrm{~dB}$ or if that is still too slow $\mathrm{TR}=0.5$, at the expense of an increased average loss of about $1.5 \mathrm{~dB}$.

\section{Tolerance to SourCe MisalignMEnt MeasuremEnTS}

To investigate the effect of source misalignment, the MM fiber of the previous experiments was replaced with a single-mode (SM) fiber and scanned along the lateral direction $x$ (Fig. 1) with the aid of the motorized stages, while monitoring the power at the other end. As an example, Fig. 3 shows the results for two scans, one for a tapered bend with TR $=0.4$ ( $w_{\text {in }}=50 \mu \mathrm{m}, w_{\text {out }}=20 \mu \mathrm{m}$ ) and $R=6 \mathrm{~mm}$ and one for the waveguide bend II with $w_{\text {in }}=w_{\text {out }}=20 \mu \mathrm{m}, R=6.5 \mathrm{~mm}$. It is clearly seen in Fig. 3 that as the fiber moves away from the position of maximum coupling efficiency (minimum insertion loss) $x=0$, the received power drops for both tapered and waveguide bends. However, although they both have the same width at their output, the tapered bend maintains high coupling efficiency for a larger range of fiber misalignment than the waveguide bend but at an increased loss penalty.

Fig. 4 compares the tapered bends with the waveguide bends by plotting the difference, $\Delta$ full-width at half-maximum (FWHM), between the FWHM obtained for a tapered bend and the FWHM obtained for the waveguide bend II in the same group, from plots like Fig. 3, as a function of taper ratio. The 


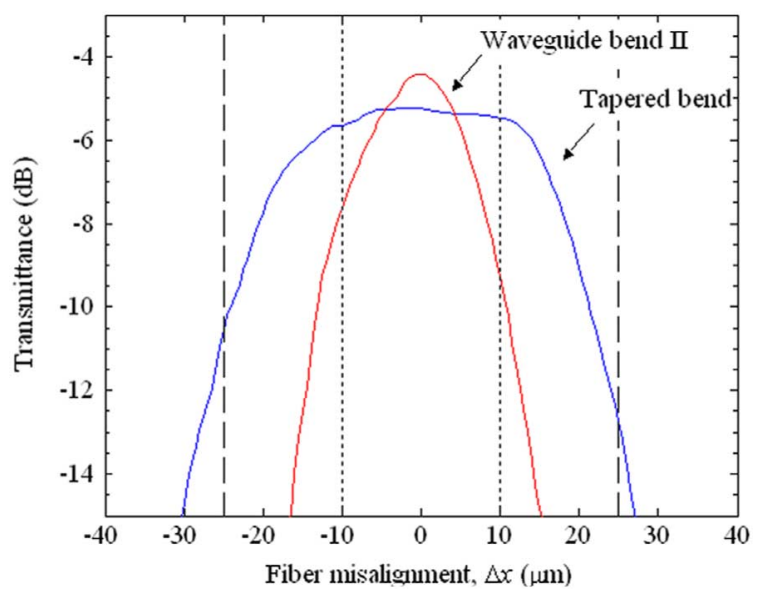

Fig. 3. Coupled power as a function of fiber lateral misalignment from the best coupling position $(\Delta x=0)$, for a tapered bend and for a waveguide bend II. Dashed lines correspond to the boundaries of the $50-\mu \mathrm{m}$-wide entrance of the tapered bend while dotted lines correspond to the boundaries of the $20-\mu \mathrm{m}$-wide entrance of the waveguide bend II. Resolution of the scans: $0.5 \mu \mathrm{m}$.

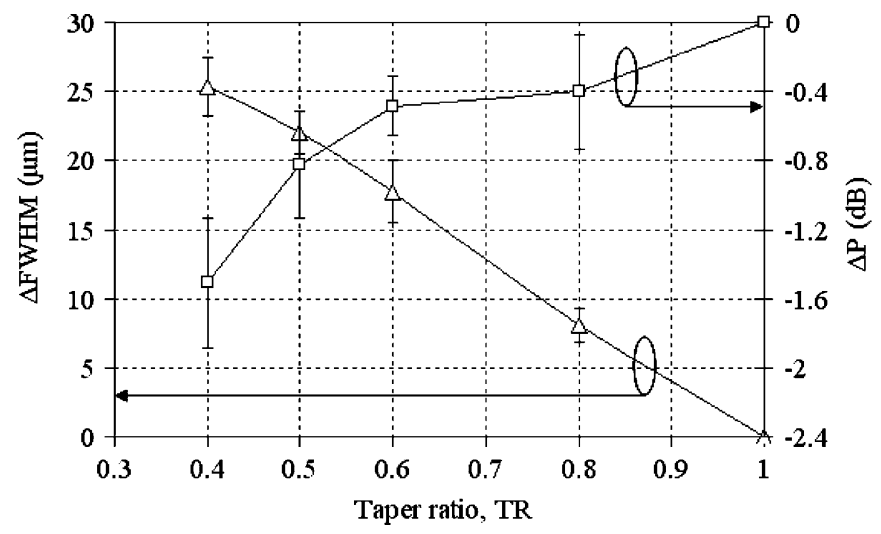

Fig. 4. Mean $\Delta \mathrm{FWHM}$ and $\Delta \mathrm{P}$ as a function of taper ratio for all tapered bends on the wafer. $\left(\mathrm{TR}=w_{\text {out }} / w_{\text {in }}\right.$.)

FWHM is the amount of misalignment that can be tolerated if the increased loss is to be less than $3 \mathrm{~dB}$. Fig. 4 also plots the difference between the power coupled in the tapered bends and the power coupled into the waveguide bends II of the same group, $\Delta P$, at best coupling position. In both cases, the means are plotted with the error-bars being the standard deviation from the six waveguides with the same TR. The positive sign of the $\Delta$ FWHM for all TR means that the tapered bend FWHM is always larger than the waveguide bend FWHM. However, the negative sign of $\Delta P$, for all TR, means that the tapered bends always have higher insertion loss than the waveguide bends.

Fig. 5 plots the product of the FWHM of the tapered bend misalignment curves in Fig. 3 times the maximum coupled power at $x=0 P$ (in linear power units this time), as a function of TR for

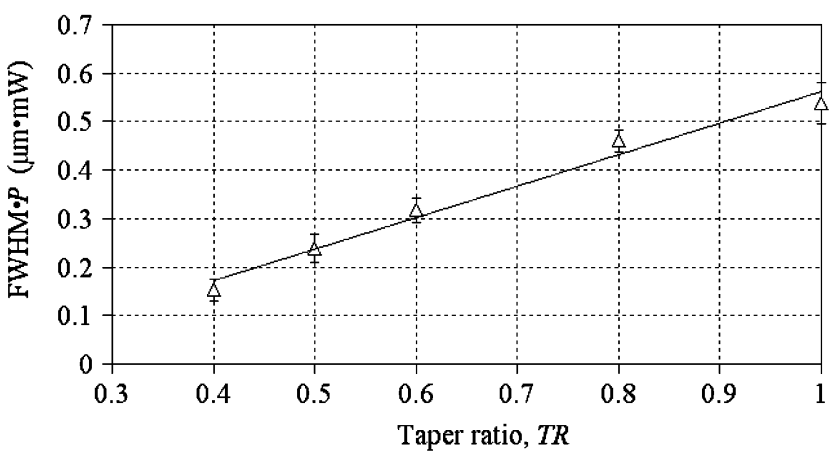

Fig. 5. Maximum coupled power times FWHM misalignment tolerance of tapered waveguide bends as a function of TR. (TR $\left.=w_{\text {out }} / w_{\text {in }}.\right)$

all radii of curvature. Again, each point is the mean FWHM $\cdot P$ product from the six waveguides with the same TR on the wafer, while the error bars are the standard deviation of these six measurements. The error bars are small, showing the FWHM. $P$ product is linearly related to the taper ratio TR and almost unrelated to the radius of curvature $R$. In this way, the effect of TR and $R$ can be separated. The effect of $R$ on loss can be found from design curves for waveguide bends [3]. The effect of TR on FWHM $P$ product can be found from a linear regression fit to Fig. 5, giving $(\mathrm{FWHM} \cdot P)=0.650 \mathrm{TRs}-0.09$.

\section{CONCLUSION}

Taper bends can provide the functions of optical path redirection and waveguide width reduction but with some penalty loss which increases as TR decreases. In addition, a direct tradeoff (in linear units) exists between the mean source misalignment tolerance and the mean best coupling efficiency in tapered waveguides. Their product is almost independent of radius of curvature of bent tapers, varying linearly with taper ratio being best for the largest taper ratio (the least steep taper).

\section{REFERENCES}

[1] L. Vivien, X. Le Roux, S. Laval, E. Cassan, and D. Marris-Morini, "Design, realization, and characterization of 3-D taper for fibre/microwaveguide coupling," IEEE J. Sel. Topics Quantum Electron., vol. 12, no. 6, pp. 1354-1358, Jun. 2006.

[2] L. Wu, F. Li, S. Tang, B. Bihari, and R. T. Chen, "Compression-molded three-dimensional tapered polymeric waveguides for low-loss optoelectronic packaging," IEEE Photon. Technol. Lett., vol. 9, no. 11, pp. 1601-1603, Nov. 1997.

[3] I. Papakonstantinou, K. Wang, D. R. Selviah, and F. A. Fernandez, "Transition, radiation and propagation loss in polymer multimode waveguide bends," Opt. Express, vol. 15, pp. 669-679, Jan. 2007.

[4] M. L. Wu, P. L. Fan, and C. T. Lee, "Completely adiabatic S-shaped bent tapers in optical waveguides," IEEE Photon. Technol. Lett., vol. 9, no. 2, pp. 212-214, Feb. 1997.

[5] C. Goyal, R. L. Gallawa, and A. K. Ghatak, "Bent planar waveguides and whispering gallery modes: A new method of analysis," J. Lightw. Technol., vol. 8, no. 5, pp. 768-774, May 1990. 